\title{
Effects of Shenkangling intervention on the MAPK pathway in rats with doxorubicin- induced nephropathy
}

\author{
S. Ai ${ }^{1,3 *}$, Y.Y. Lin ${ }^{4 *}$, J. Zheng, ${ }^{2,3}$, C.X. Qiu' ${ }^{1}$, Y.J. Liu ${ }^{1}$ and X. Lin ${ }^{1}$ \\ ${ }^{1}$ People's Hospital Affiliated to Fujian University of Traditional Chinese Medicine, \\ Fuzhou, Fujian, China \\ ${ }^{2}$ Fujian University of Traditional Chinese Medicine, Fuzhou, Fujian, China \\ ${ }^{3}$ Key Laboratory of Integrative Kidney Disease in Fujian Province, \\ Fuzhou, Fujian, China \\ ${ }^{4}$ Fuzhou General Hospital of Nanjing Military District, Fuzhou, Fujian, China \\ *These authors contributed equally to this study. \\ Corresponding author: J. Zheng \\ E-mail: zjiandr@163.com
}

Genet. Mol. Res. 15 (3): gmr.15038131

Received November 25, 2015

Accepted January 15, 2016

Published August 18, 2016

DOI http://dx.doi.org/10.4238/gmr.15038131

Copyright (C) 2016 The Authors. This is an open-access article distributed under the terms of the Creative Commons Attribution ShareAlike (CC BY-SA) 4.0 License

\begin{abstract}
Shenkangling plays a role of Yishenhuoxue effect for the treatment of children with nephrotic syndrome. The aim of this study was to investigate the effects of Shenkangling intervention on the mitogen-activated protein kinase (MAPK) pathway in rats with Adriamycin-induced nephropathy (AN) and its underlying mechanism of action. Nephrosis was induced in healthy Sprague-Dawley rats by doxorubicin and the rats were untreated or treated with prednisone, simvastatin, Shenkangling, or a combination thereof. Using real-time PCR, the mRNA expression levels of Chemokine (C-X-C motif) ligand 16 (CXCL16), A Disintegrin and metalloproteinase domain-
\end{abstract}


containing protein 10 (ADAM10), and ADAM17 in the renal tissues of these rats were found to be decreased by the various treatments compared to those in the untreated doxorubicin-induced nephrosis rats. To quantify the activation of the MAPK pathway, western blotting was used to detect the phosphorylation levels of MAPK pathwayassociated proteins (p38, ERK1/2, SAPK/JNK) and nuclear factor (NF)- $\mathrm{KB}$ p65, which were reduced by the various treatments compared to those in the untreated doxorubicin-induced rats. Serum levels of transforming growth factor (TGF)- $\beta 1$, tumor necrosis factor (TNF)- $\alpha$, interleukin (IL)-1 $\beta$, and IL-6, quantified by ELISA, were decreased by the various treatments compared to the levels in the untreated doxorubicin-induced nephrosis rats. The rats treated with prednisone, simvastatin, and Shenkangling showed the best outcome. The Chinese medicine Shenkangling that is known for nourishing the kidney and promoting blood circulation reduced urinary protein levels, increased serum albumin levels, and reduced cholesterol levels by reducing the release of CXCL16, ADAM10, ADAM17, TGF- $\beta 1$, TNF- $\alpha$, IL-1 $\beta$, IL6 , and other inflammatory mediators and inhibiting the activation of the MAPK signaling pathway, thereby effectively improving the state of nephropathy in AN rats. These results indicate that Shenkangling can be used clinically to treat nephropathy.

Key words: MAPK pathway; Doxorubicin; Shenkangling

\section{INTRODUCTION}

Primary nephrotic syndrome (PNS) is a common glomerular disease occurring in childhood. It has been reported in foreign countries that the annual incidence of PNS was 2-4 of 100,000 children, whereas the prevalence was 16 of 100,000 children (Eddy and Symons, 2003; Wong, 2007). In some of China's provinces and municipalities, PNS accounted for 21 to $31 \%$ of hospitalized pediatric urologic diseases. More than $85 \%$ of the children with PNS had a pathological type of minimal change, most of whom were sensitive to glucocorticoids. In children with PNS, 80 to $90 \%$ of the initial hormone therapy may result in complete remission, but 76 to $93 \%$ of the treatment cases can relapse. Of the relapse cases, 45 to $50 \%$ were frequent occurrences or steroid-dependent (Koskimies et al., 1982; Tarshish et al., 1997). The doxorubicin-induced nephrosis rat model has been widely used in PNS experimental research since the early 1980s; this acute model of pathological changes are similar to human. It has also been reported that changes of clinical histology and experimental performance in AN rats were similar to kidney deficiency and blood stasis (Li and Wang, 2002; Liang, 2005; Li and Zhang, 2006). Kidney nourishment and blood circulation activation can significantly improve symptoms and indicators for AN rats.

The aim of this study was to analyze changes in the mitogen-activated protein kinase (MAPK) signaling pathway in the kidney tissues of AN rats treated with Shenkangling, and to further clarify the mechanism of Shenkangling intervention in children with PNS at the molecular and genetic levels, providing experimental evidence for the clinical application of Shenkangling, which plays a role of Yishenhuoxue effect for the treatment of children with nephrotic syndrome.

Genetics and Molecular Research 15 (3): gmr.15038131 


\section{MATERIAL AND METHODS}

\section{Experimental materials}

\section{Animal source}

Healthy male Sprague-Dawley rats $(\mathrm{N}=75), 2-3$ months old, weighing 200-300 g, were provided by Shanghai SLRC Laboratory Animal Co., with Certificate of Conformity: SCXK (Shanghai) 2007-0005.

\section{Experimental drugs}

Shenkangling was prepared by boiling, filtering, centrifuging, and concentrating $30 \mathrm{~g}$ Astragalus, $15 \mathrm{~g}$ Rehmannia, $9 \mathrm{~g}$ dogwood, $12 \mathrm{~g}$ yam, $9 \mathrm{~g}$ poria, $15 \mathrm{~g}$ moutan, and $9 \mathrm{~g}$ pseudoginseng. The crude drug concentration was $1.5 \mathrm{~g} / \mathrm{mL}$ [drug concentration was calculated by referring to the Animal Experimental Methodology (Sun, 2001), approved by the preparation room of the People's Hospital Affiliated to Fujian University of Traditional Chinese Medicine]. Doxorubicin hydrochloride for injection was provided by Pharmacia \& Upjohn (Peapack, NJ, USA), batch number: 0906E2. Prednisone, $5 \mathrm{mg} /$ tablet, was purchased from Shanghai Xinyi Pharmaceutical (Shanghai, China), lot number 018130 302. Simvastatin, $20 \mathrm{mg}$ /tablet, was purchased from Guangzhou Nanxin Pharmaceutical (Guangzhou, China), lot number 3137152.

\section{Main kits and equipment}

Trizol and PCR primers for Chemokine (C-X-C motif) ligand 16 (CXCL16), A Disintegrin and metalloproteinase domain-containing protein 10 (ADAM10), and ADAM17 purchased from Invitrogen (Carlsbad, CA, USA). RevertAid ${ }^{\mathrm{TM}}$ First-Strand cDNA Synthesis Kit was purchased from Fermentas (Waltham, MA, USA). ReverTraAce ${ }^{\circledR} \mathrm{qPCR}$ RT Kit was purchased from TOYOBO (Shanghai, China). SYBR ${ }^{\circledR}$ Premix Ex Taq ${ }^{\mathrm{TM}}$ II was purchased from TaKaRa (Dalian, China). Phosphorylation-specific antibodies for p38, extracellular signal-regulated kinase (ERK)1/2, stress-activated protein kinase/c-Jun N-terminal kinase (SAPK/JNK), and nuclear factor-kappa B (NF-kB) p65 were purchased from Cell Signaling Technology (Danvers, MA, USA), with product codes $9212 \mathrm{~S}, 9102 \mathrm{~S}, 9258 \mathrm{~S}$, and 3031S, respectively. Transforming growth factor (TGF)- $\beta 1$, tumor necrosis factor (TNF)- $\alpha$, interleukin (IL)-1 $\beta$, and IL-6 antibodies were purchased from Shanghai Weiao Biotech (Shanghai, China), with product codes ER0152, ER0155, ER0128, and ER0132, respectively. The PCR instrument (model: PTC-225 Peltier Thermal Cycler) was provided by MJ Research (Waltham, MA, USA). The fluorescence quantitative PCR instrument (model: CFX-96) was provided by Bio-Rad (Hercules, CA, USA). The gel imaging system (model: Tanon 2500) was purchased from Tanon (Shanghai, China).

\section{Experimental methods and procedures}

\section{Grouping}

According to the body mass stratified random principle, rats were divided into the

Genetics and Molecular Research 15 (3): gmr.15038131 
control group, AN model group, prednisone treatment group, prednisolone + Shenkangling treatment group, prednisone + simvastatin treatment group, and prednisone + simvastatin + Shenkangling treatment group, at 10 rats per group.

\section{Recapitulating AN model}

On day 1, doxorubicin was injected disposably and intravenously in the rats in all the groups except for the control group $(5.5 \mathrm{mg} / \mathrm{kg}$ body weight $)$, while equivalent doses of saline were injected in the control rats in the same manner.

\section{Methods of drug administration}

Starting on day 11 after the one-time intravenous injection of doxorubicin, rats of the control and AN model groups received $3 \mathrm{~mL}$ distilled water via the stomach. The rats of the prednisone group were administered prednisone suspension $(5.5 \mathrm{mg} / \mathrm{kg}$ body weight per day) via the stomach in the mornings, and $3 \mathrm{~mL}$ distilled water in the afternoons. The rats of the prednisone + Shenkangling group were administered prednisone suspension via the stomach in the mornings, and the Shenkangling decoction at $1 \mathrm{~mL} / 100 \mathrm{~g}$ body weight in the afternoons. The rats of the prednisone + simvastatin group were administered prednisone suspension via the stomach in the mornings, and simvastatin suspension in the afternoons ( $5 \mathrm{mg} / \mathrm{kg}$ body weight per day). The rats of the prednisone + simvastatin + Shenkangling group were administered prednisone suspension via the stomach in the mornings, simvastatin at noon, and the Shenkangling decoction in the afternoons. Distilled water was the solvent of the prednisone and simvastatin suspensions. All rats received intragastric administration for 22 days.

\section{Twenty-four-hour urinary protein detection}

Prior to the injection of doxorubicin, on day 10 after doxorubicin injection (one day before treatment), 1 week after treatment, 2 weeks after treatment, and 3 weeks after treatment, the rats were fed in a separate metabolic cage and urine samples were collected for $24 \mathrm{~h}$. The amount of urine was recorded, and the $4 \%$ salicylic acid assay was used to detect 24 -h urinary protein levels.

\section{Detection of blood biochemical parameters}

At the end of the experiment, blood samples were drawn from the abdominal aorta of all rats and were coagulated for $2 \mathrm{~h}$ at room temperature or overnight at $2-8^{\circ} \mathrm{C}$. The samples were centrifuged at $1500 \mathrm{~g}$ for $15 \mathrm{~min}$ and the sera were collected. Serum albumin, cholesterol, and other indicators were measured using an automatic biochemical analyzer. The remaining serum samples were preserved at $-20^{\circ} \mathrm{C}$ for further experiments.

\section{ELISA assay to detect serum levels of TGF- $1, T N F-\alpha, I L-1 \beta$, and IL-6 in rats}

The serum samples were loaded onto a microtiter plate, lid was added, and samples were reacted at $37^{\circ} \mathrm{C}$ for $90 \mathrm{~min}$. After reaction, an automatic washer was used either to absorb 
the liquid within the microtiter plate, or to reject the liquid within the microtiter plate, and then shoot against absorbent paper several times. The biotinylated anti-rat TGF- $\beta 1$, TNF- $\alpha$, IL- $1 \beta$, and IL- 6 antibody solutions were added at increasing concentrations with $0.1 \mathrm{~mL}$ per well, excluding tetramethylbenzidine (TMB), blank-coloring wells. The mixtures were reacted at $37^{\circ} \mathrm{C}$ for $60 \mathrm{~min}$. The wells were washed three times with $0.01 \mathrm{M}$ Tris-buffered solution (TBS) or $0.01 \mathrm{M}$ phosphate-buffered solution (PBS), soaking 1 min each time. Avidin-biotinpcroxidase complex $(\mathrm{ABC})$ was prepared and added at $0.1 \mathrm{~mL}$ per well (TMB was left blank except for the color well). The mixtures were further reacted at $37^{\circ} \mathrm{C}$ for $30 \mathrm{~min}$, and washed five times with $0.01 \mathrm{M}$ TBS or $0.01 \mathrm{M}$ PBS, soaking for about 1-2 min each time. TMB color solution, which had been balanced at $37^{\circ} \mathrm{C}$ for $30 \mathrm{~min}$, was added successively at $90 \mu \mathrm{L}$ per well. The wells were reacted at $37^{\circ} \mathrm{C}$ in the dark for $25 \mathrm{~min}$. TMB-ending solution at $0.1 \mathrm{~mL}$ per well was added, and the color of the wells changed from blue to yellow. Optical density values were measured at $450 \mathrm{~nm}$ by a microplate reader.

\section{Real time-PCR to detect gene levels of CXCL16, ADAM10, and ADAM17 in rat renal tissue}

Total RNA extraction and RT-PCR were performed as previously described (Wan et al., 2014).

\section{Western blot assay to detect phosphorylation levels of p38, ERK1/2, SAPK/JNK and $N F-\kappa B$ p65 in rat renal tissue}

About 300 g kidney tissue was milled, and $100 \mu \mathrm{L}$ cold RIPA buffer [each milliliter containing $5 \mu \mathrm{L} 100 \mathrm{mM}$ phenylmethanesulfonyl fluoride (PMSF), and $5 \mu \mathrm{L}$ protease inhibitors] was added to each sample. After being vortexed for $15 \mathrm{~s}$, the samples were lysed on ice for $10 \mathrm{~min}$; this procedure was repeated four times. Total protein was extracted from each sample for western blotting, performed as previously described (Xing et al., 2014).

\section{Statistical analysis}

The SPSS13.0 statistical software was used for analysis; data are reported as means \pm $\mathrm{SD}$. Differences between two groups were compared using one-way ANOVA analysis, and a P value of less than 0.05 was considered statistically significant.

\section{RESULTS}

\section{Urinary protein quantitation (24 h)}

In the $\mathrm{AN}$ rats without treatment, 24-h urinary protein levels significantly increased $(\mathrm{P}<0.01)$; each treatment significantly reduced urinary protein levels $(\mathrm{P}<0.01)$. There was no significant difference between the treatment groups in the first week and second week $(\mathrm{P}>0.05)$. However, in the third week, urine protein levels in the prednisone + simvastatin + Shenkangling group were lower than those in the prednisone group, the prednisolone + Shenkangling group, and the prednisone + simvastatin group $(\mathrm{P}<0.01$; Table 1$)$. 
Table 1. Rat urinary protein quantitation $(\mathrm{g} / 24 \mathrm{~h})$.

\begin{tabular}{l|c|c|c}
\hline Groups & 1st week & 2nd week & 3rd week \\
\hline Control & $5.25 \pm 1.66$ & $5.15 \pm 1.48$ & $5.17 \pm 1.63$ \\
\hline AN model & $121.60 \pm 22.93^{* \#}$ & $129.60 \pm 22.00^{* \#}$ & $149.56 \pm 23.05^{* \#}$ \\
\hline Prednisone & $104.51 \pm 10.47^{* \Delta \#}$ & $96.11 \pm 9.98^{* \Delta \#}$ & $78.37 \pm 11.36^{* \Delta \#}$ \\
\hline Prednisone + Shenkangling & $104.45 \pm 14.64^{* \Delta \#}$ & $90.78 \pm 7.40^{* \Delta \#}$ & $73.97 \pm 9.23^{* \Delta \#}$ \\
\hline Prednisone + simvastatin & $98.90 \pm 10.68^{* \Delta \#}$ & $92.99 \pm 12.76^{* \Delta \#}$ & $71.26 \pm 11.97^{* \Delta \#}$ \\
\hline Prednisone + simvastatin + Shenkangling & $95.14 \pm 5.48^{* \Delta}$ & $85.48 \pm 14.36^{* \Delta}$ & $45.98 \pm 11.80^{* \Delta}$ \\
\hline
\end{tabular}

$\mathrm{N}=10$ in each group. $* \mathrm{P}<0.01$, compared with the control group; ${ }^{\Delta} \mathrm{P}<0.01$, compared with the $\mathrm{AN}$ model group; ${ }^{*} \mathrm{P}<0.01$, compared with the prednisone + simvastatin + Shenkangling group.

\section{Serum albumin and cholesterol levels}

Serum albumin levels in AN rats were significantly lower $(\mathrm{P}<0.01)$, while cholesterol levels were significantly higher $(\mathrm{P}<0.01)$ than those in the control group. Albumin levels significantly increased and cholesterol levels significantly reduced in each treatment group when compared to the AN group $(\mathrm{P}<0.01)$. The effect was the most prominent in the prednisone + simvastatin + Shenkangling group $(\mathrm{P}<0.01$ or $\mathrm{P}<0.05$; Table 2$)$.

\section{Table 2. Serum albumin and cholesterol levels.}

\begin{tabular}{l|c|c|c}
\hline Groups & $\mathrm{N}$ & Albumin $(\mathrm{g} / \mathrm{L})$ & Cholesterol $(\mathrm{mM})$ \\
\hline Control & 10 & $40.71 \pm 3.55$ & $1.67 \pm 0.22^{*}$ \\
\hline AN model & 10 & $18.02 \pm 4.30^{*}$ & $11.32 \pm 4.16^{*}$ \\
\hline Prednisone & 10 & $27.01 \pm 4.29^{* \triangle \#}$ & $7.61 \pm 2.12^{* \Delta \#}$ \\
\hline Prednisone + Shenkangling & 10 & $27.95 \pm 3.94^{* \Delta \#}$ & $6.94 \pm 1.63^{* \Delta \#}$ \\
\hline Prednisone + simvastatin & 10 & $27.70 \pm 4.98^{* \# \#}$ & $6.39 \pm 0.70^{* \Delta \#}$ \\
\hline Prednisone + simvastatin + Shenkangling & 10 & $32.15 \pm 2.49^{* \Delta}$ & $4.39 \pm 0.80^{* \Delta}$ \\
\hline
\end{tabular}

${ }_{* \mathrm{P}}<0.01$, compared with the control group; ${ }^{\Delta} \mathrm{P}<0.01$, compared with the AN model group; ${ }^{\#} \mathrm{P}<0.01,{ }^{\#} \mathrm{P}<0.05$, compared with the prednisone + simvastatin + Shenkangling group.

\section{Serum levels of TGF- $\beta 1$, TNF- $\alpha$, IL-1 $\beta$, and IL-6}

Serum levels of TGF- $\beta 1$, TNF- $\alpha$, IL-1 $\beta$, and IL-6 in AN rats were significantly higher than those in the control group (all $\mathrm{P}<0.01$ ), and significantly lower in each treatment group than in the AN group (all $\mathrm{P}<0.01$ ). The prednisone + simvastatin + Shenkangling treatment was better than the prednisone, prednisolone + Shenkangling, and prednisone + simvastatin treatments in reducing the levels of TGF- $\beta 1$, TNF- $\alpha$, IL- $1 \beta$, and IL-6 $(\mathrm{P}<0.01$ or $\mathrm{P}<0.05)$, as shown in Table 3.

\footnotetext{
Table 3. Serum levels of TGF- $\beta 1$, TNF- $\alpha$, IL-1 $\beta$, and IL-6 (pg/mL).

\begin{tabular}{|c|c|c|c|c|c|}
\hline Groups & $\mathrm{N}$ & TGF- $\beta 1$ & TNF- $\alpha$ & IL-1 $\beta$ & IL-6 \\
\hline Control & 10 & $5219.3 \pm 1759.05$ & $2.63 \pm 1.35$ & $3.46 \pm 2.41$ & $5.53 \pm 1.09$ \\
\hline AN model & 10 & $22145.06 \pm 4705.60^{*}$ & $26.27 \pm 11.02^{*}$ & $267.56 \pm 54.38^{*}$ & $252.48 \pm 49.02^{*}$ \\
\hline Prednisone & 10 & $15145.35 \pm 4002.61^{* \triangle 4}$ & $15.74 \pm 1.00^{* \Delta \#}$ & $122.70 \pm 31.78^{* \Delta H}$ & $99.29 \pm 9.41^{* \Delta *}$ \\
\hline Prednisone + Shenkangling & 10 & $15023.18 \pm 2271.35^{* \triangle A}$ & $12.75 \pm 0.96^{* \Delta * A+}$ & $122.55 \pm 27.45^{* \Delta *}$ & $97.87 \pm 10.04^{* \Delta *}$ \\
\hline Prednisone + simvastatin & 10 & $16695.51 \pm 2268.86^{* \triangle 4}$ & $12.51 \pm 1.72^{* \Delta \# H}$ & $116.96 \pm 22.99^{* \Delta \#}$ & $84.10 \pm 10.04^{* \Delta A}$ \\
\hline Prednisone + simvastatin + Shenkangling & 10 & $7414.31 \pm 1778.81^{* \Delta}$ & $7.32 \pm 1.56^{* \Delta}$ & $55.49 \pm 11.3^{* \Delta}$ & $42.00 \pm 16.26^{* \Delta}$ \\
\hline
\end{tabular}

$* \mathrm{P}<0.01$, compared with the control group; ${ }^{\Delta} \mathrm{P}<0.01$, compared with the AN model group; ${ }^{\#} \mathrm{P}<0.01,{ }^{\#} \mathrm{P}<0.05$, compared with the prednisone + simvastatin + Shenkangling group.
} 


\section{Gene levels of CXCL16, ADAM10, and ADAM17 in kidney tissues}

mRNA levels of CXCL16, ADAM10, and ADAM17 in AN rats were significantly higher than those in the control group (all $\mathrm{P}<0.01$ ), and significantly lower in each treatment group than in the AN model group (all $\mathrm{P}<0.01$ ). The prednisone + simvastatin + Shenkangling treatment was better than the prednisone, prednisolone + Shenkangling, and prednisone + simvastatin treatments in reducing the gene levels of CXCL16, ADAM10, and ADAM17 (P < 0.01 or $\mathrm{P}<0.05$ ), as shown in Table 4 .

\section{Table 4. Gene levels of CXCL16, ADAM10, and ADAM17 in kidney tissues.}

\begin{tabular}{l|c|c|c|c}
\hline Groups & $\mathrm{N}$ & CXCL16 & ADAM10 & ADAM17 \\
\hline Control & 10 & $0.03 \pm 0.01$ & $0.03 \pm 0.01$ & $0.02 \pm 0.01$ \\
\hline AN model & 10 & $4.27 \pm 1.45^{*}$ & $5.15 \pm 1.59^{*}$ & $6.07 \pm 1.57^{*}$ \\
\hline Prednisone & 10 & $2.77 \pm 1.20^{* \triangle \#}$ & $3.16 \pm 1.19^{* \Delta \#}$ & $3.65 \pm 1.10^{* \Delta \#}$ \\
\hline Prednisone + Shenkangling & 10 & $1.96 \pm 1.00^{* \star \# \#}$ & $3.09 \pm 1.20^{* \triangle \#}$ & $3.68 \pm 1.24^{* \triangle \#}$ \\
\hline Prednisone + simvastatin & 10 & $1.94 \pm 0.73^{* \triangle \#}$ & $2.92 \pm 0.74^{*} \triangle \#$ & $3.56 \pm 1.28^{* \Delta \#}$ \\
\hline Prednisone + simvastatin + Shenkangling & 10 & $0.99 \pm 0.66^{* \Delta}$ & $1.64 \pm 1.08^{* \Delta}$ & $1.62 \pm 0.86^{* \Delta}$ \\
\hline
\end{tabular}

${ }^{*} \mathrm{P}<0.01$, compared with the control group; ${ }^{\Delta} \mathrm{P}<0.01$, compared with the AN model group; ${ }^{\#} \mathrm{P}<0.01,{ }^{\# \#} \mathrm{P}<0.05$, compared with the prednisone + simvastatin + Shenkangling group.

\section{Phosphorylation levels of MAPK pathway proteins in renal tissues}

Phosphorylation levels of p38, ERK1/2, SAPK/JNK, and NF- $\mathrm{BB}$ p65 in AN rats were significantly higher than in the control group (all $\mathrm{P}<0.01$ ), and significantly reduced in each treatment group compared to the $\mathrm{AN}$ model group (all $\mathrm{P}<0.01$ ). The prednisone + simvastatin + Shenkangling treatment was better than the prednisone, prednisolone + Shenkangling, and prednisone + simvastatin treatments in reducing the phosphorylation levels of p38, ERK1/2,

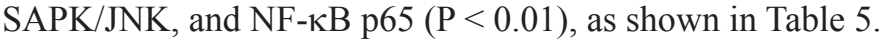

Table 5. Phosphorylation levels of the MAPK pathway in rat renal tissue.

\begin{tabular}{|c|c|c|c|c|c|}
\hline Groups & $\mathrm{N}$ & Phospho-p38 & Phospho-ERK1/2 & Phospho-SAPK/JNK & Phospho-NF-kB p65 \\
\hline Control & 10 & $2667.06 \pm 636.09$ & $2594.79 \pm 740.18$ & $3111.13 \pm 694.01$ & $2391.10 \pm 340.67$ \\
\hline AN model & 10 & $19406.30 \pm 3643.29^{*}$ & $20023.23 \pm 3475.89^{*}$ & $19522.40 \pm 3449.96^{*}$ & $18900.90 \pm 796.68^{*}$ \\
\hline Prednisone & 10 & $8894.90 \pm 937.07 * \Delta *$ & $9252.81 \pm 821.32^{* \Delta \#}$ & $9700.30 \pm 1275.06^{* \Delta H}$ & $9578.10 \pm 1085.00^{* \Delta \#}$ \\
\hline Prednisone + Shenkangling & 10 & $8688.72 \pm 737.26^{* \Delta \#}$ & $8890.19 \pm 964.02^{* \Delta H}$ & $9143.19 \pm 1100.39^{* \Delta *}$ & $9099.00 \pm 635.45^{* \triangle H}$ \\
\hline Prednisone + simvastatin & 10 & $9024.00 \pm 798.18^{* \Delta 4}$ & $8992.10 \pm 802.77^{* \Delta \#}$ & $9127.16 \pm 360.87^{* \Delta *}$ & $9225.00 \pm 981.66^{* \triangle 4}$ \\
\hline Prednisone + simvastatin + Shenkangling & 10 & $4713.42 \pm 646.73^{* \Delta}$ & $4805.93 \pm 646.48^{* \Delta}$ & $4593.10 \pm 568.31^{* \Delta}$ & $4569.60 \pm 791.72 * \Delta$ \\
\hline
\end{tabular}

$* \mathrm{P}<0.01$, compared with the control group; ${ }^{\Delta} \mathrm{P}<0.01$, compared with the AN model group; ${ }^{\#} \mathrm{P}<0.01$, compared with the prednisone + simvastatin + Shenkangling group.

\section{DISCUSSION}

Our AN model rats had typical manifestations of nephrotic syndrome: proteinuria, hypoalbuminemia, and hyperlipidemia, prompting the release of CXCL16, ADAM10, ADAM17, TGF- $\beta 1$, TNF- $\alpha$, IL- $1 \beta$, IL- 6 , and other inflammatory mediators to activate the MAPK signaling pathway (p38, ERK1/2, SAPK/JNK) and increase the activity of NF- $\kappa B$ p65, triggering a cascade of immune responses, further damaging glomeruli. The traditional Chinese medicine Shenkangling, which can nourish the kidneys and activate blood circulation, reduced the activity of NF- $\mathrm{kB}$ p 65 by inhibiting the release of the above-mentioned inflammatory 
mediators and inhibiting the activation of the MAPK signaling pathway, reducing the urinary protein levels of AN rats, increasing serum albumin levels, and reducing cholesterol levels, thereby effectively improving the nephropathy state of AN rats. This study lays a foundation for improving the clinical efficacy of pediatric PNS and clinical application of kidney nourishing and blood circulation-activating decoction, and provides novel ideas and methods for the clinical study of traditional Chinese medicine to delay glomerulosclerosis.

Currently, prednisone is still the first choice of treatment for PNS in China and abroad, or in combination with immunosuppressive agents. Although prednisone can alleviate some PNS cases and reduce the relapse rate, the incidence of side effects of hormones and immunosuppressants can also increase. Long-term use of large doses of hormones can also lead to adrenal insufficiency, which is a main reason for frequent episodes of PNS. Clinical hormones are often seen as Okara hot products by herbalist doctors, and produce heat to reinforce yang and reduce body fluid consume. Clinical studies have shown that the hypothalamic-pituitaryadrenal axis is closely related to the kidney in Chinese medicine; the syndrome differentiation proceeding from the kidney and combining with clinical manifestations is the common basis for the treatment of kidney disease in Chinese medicine.

We have found that in past clinical studies and animal experiments, tonifying kidney Chinese medicine had a similar effect as corticosteroids, which can reduce feedback inhibition of exogenous hormones on the adrenal cortex, preventing the failure of pituitary adrenal cortex and regulating the body's immune function to reduce the infection rate, relapse rate, and side effects in children with PNS. Traditional Chinese medicine of activating blood circulation to dissipate blood stasis can promote the activation of the monocyte-macrophage system, suppress specific immunity, reduce capillary permeability, improve renal microcirculation, reduce inflammatory renal tissue damage, and inhibit platelet function, thereby promoting fibrinolysis, which can also reduce structural tissue hyperplasia and promote tissue repair. The methods of kidney nourishment and blood activation mutually collaborate to improve the pathological changes of kidney deficiency and blood stasis by regulating the function of nervous, endocrine, and immune systems and improving microcirculation. These results have been demonstrated in preliminary animal experiments and clinical studies (Zheng et al., 2004a,b, 2005, 2008).

CXCL16 is an oxidized low-density lipoprotein (ox-LDL) cell surface receptor first studied in the early 21 st century; it has a strong ox-LDL-binding activity and endocytic uptake ability, participating in relevant biological effects. It is known as a scavenger receptor that binds phosphatidylserine and oxidized lipoproteins (Mitsuoka et al., 2009). Garcia et al. (2007) found that in glomerular nephritis, CXCL16 was expressed in glomerular endothelial cells under the stimulation of pro-inflammatory factors, while IL-1 $\beta$ promoted CXCL16 expression in mesangial cells. Inhibiting the expression of CXCL16 can weaken anti-glomerular basement membrane antibody-mediated glomerulonephritis; its occurrence may be related to the strong chemotactic effects of CXCL16 on glomerular infiltrated granulocytes. ADAM10 and ADAM17 are widely expressed in most organisms, with some differences between species, but with similar functions, which is to shed transmembrane molecules. CX3CLl is a transmembrane-inducing factor, and when bound to the receptor CX3CR1, can induce cell-cell adhesion. In the context of ADAM10 and ADAM17, CX3CL1 releases soluble cell-inducing factors to induce the aggregation of granulocytes. The overexpression of ADAM10 may promote CX3CLl hydrolysis, thus modulating the interaction of monocytes and the cell layers

Genetics and Molecular Research 15 (3): gmr.15038131 
expressing CX3CLI (Ko et al., 2007), and promoting the separation of monocytes from cell substrate. The results of this study indicated that ADAM10 and ADAM17 had a significant correlation with CXCL16, so ADAM10 and ADAM17 may be involved in mediating the shedding of CXCL16 to induce inflammatory immune response and increase the mesangial matrix, which can increase glomerular injury and gradually develop into glomerular fibrosis and sclerosis, forming an irreversible trend.

MAPK is a class of widespread intracellular serine/threonine protein kinases. Studies have confirmed that the MAPK signaling pathways are mainly distributed in the cytoplasm and can be activated by a variety of stimulating factors such as cytokines, growth factors, neurotransmitters, hormones, cell stress proteins, and cell adhesion proteins. Through further signaling transduction, transcription factor activity is activated to regulate the expression of proteins, ultimately leading to cellular reactions such as cell proliferation, differentiation, conversion, and apoptosis. Experimental studies have shown that in patients with diabetic nephropathy, p38-MAPK activity changed significantly (Wu et al., 2012). In the study by Wu et al. (2012), rat glomerular mesangial cells were incubated with different concentrations of glucose as the osmotic pressure control. High sugar and hypertonic conditions could lead to the decrease in the expression of matrix metalloproteinase 9 (MMP-9) mRNA in rat glomerular mesangial cells, and the increase in mRNA expression of tissue inhibitors of matrix metalloproteinase (TIMP-1). With the increase of glucose concentration, the change was more obvious. Further, p38-MAPK-specific inhibitor SB203580 was used to block p38MAPK activity and the expression changes of p38-MAPK, MMP-9, and TIMP-1 mRNA were observed to confirm that high glucose regulated the expression of MMP-9 and TIMP-1 mRNA through the p38-MAPK signaling pathway.

These results indicate that Shenkangling can be used clinically to treat nephropathy.

\section{Conflicts of interest}

The authors declare no conflicts of interest.

\section{ACKNOWLEDGMENTS}

Research supported by the National Natural Science Foundation of China (\#81202835 and \#81173434).

\section{REFERENCES}

Eddy AA and Symons JM (2003). Nephrotic syndrome in childhood. Lancet 362: 629-639. http://dx.doi.org/10.1016/ S0140-6736(03)14184-0

Garcia GE, Truong LD, Li P, Zhang P, et al. (2007). Inhibition of CXCL16 attenuates inflammatory and progressive phases of anti-glomerular basement membrane antibody-associated glomerulonephritis. Am. J. Pathol. 170: 14851496. http://dx.doi.org/10.2353/ajpath.2007.060065

Ko SY, Lin SC, Wong YK, Liu CJ, et al. (2007). Increase of disintergin metalloprotease 10 (ADAM10) expression in oral squamous cell carcinoma. Cancer Lett. 245: 33-43. http://dx.doi.org/10.1016/j.canlet.2005.10.019

Koskimies O, Vilska J, Rapola J and Hallman N (1982). Long-term outcome of primary nephrotic syndrome. Arch. Dis. Child. 57: 544-548. http://dx.doi.org/10.1136/adc.57.7.544

Li CS and Zhang ZZ (2006). Effect of nephrosis II formula on doxorubicin nephrosis model rats' serum tumor necrosin, endotheliolysin and blood-lipid. Zhe Jiang Zhong Yi Yao Da Хие Хие Bao 30: 358-362.

Genetics and Molecular Research 15 (3): gmr.15038131 
Li Y and Wang C (2002). The effect of Bao Shenkang on hemodynamics of doxorubicin-induced nephrotic rats. Liao Ning Zhong Yi Xue Yuan Xue Bao 4: 322-324.

Liang RQ (2005). The experimental study on the kidney and spleen promoting blood circulation prescription on doxorubicin induced nephropathy in rats. Xin Zhong Yi 37: 94-95.

Mitsuoka H, Toyohara M, Kume N, Hayashida K, et al. (2009). Circulating soluble SR-PSOX/CXCL16 as a biomarker for acute coronary syndrome -comparison with high-sensitivity C-reactive protein. J. Atheroscler. Thromb. 16: 586593. http://dx.doi.org/10.5551/jat.1081<bok $>$ Sun JF (2001). Animal Experimental Methodology, Beijing, People's Health Publishing House: $357 .</$ bok $>$

Tarshish P, Tobin JN, Bernstein J and Edelmann Jr CM (1997). Prognostic significance of the early course of minimal change nephrotic syndrome: report of the International Study of Kidney Disease in Children. J. Am. Soc. Nephrol. 8: 769-776.

Wan X, Xia W, Gendoo Y, Chen W, et al. (2014). Upregulation of stromal cell-derived factor 1 (SDF-1) is associated with macrophage infiltration in renal ischemia-reperfusion injury. PLoS One 9: e114564. http://dx.doi.org/10.1371/ journal.pone. 0114564

Wong W (2007). Idiopathic nephrotic syndrome in New Zealand children, demographic, clinical features, initial management and outcome after twelve-month follow-up: results of a three-year national surveillance study. $J$. Paediatr. Child Health 43: 337-341. http://dx.doi.org/10.1111/j.1440-1754.2007.01077.x

Wu Z, Xiong Y, Gajanayake T, Ming XF, et al. (2012). p38 Mitogen-activated protein kinase is required for glucosamineinduced endothelial nitric oxide synthase uncoupling and plasminogen-activator inhibitor expression. Circ. J. 76: 2015-2022. http://dx.doi.org/10.1253/circj.CJ-12-0016

Xing LN, Wang H, Yin PH, Liu YJ, et al. (2014). Reduced mir-29b-3p expression up-regulate CDK6 and contributes to IgA nephropathy. Int. J. Clin. Exp. Med. 7: 5275-5281.

Zheng J, Wu QL, Zheng JX, Zeng ZC, et al. (2004a). Shenkangling on adriamycin induced nephropathy rat thromboxane B_2 and 6-keto prostaglandin F_(1 alpha) effect. Zhong Yi Za Zhi 45: 619-622.

Zheng J, Wu QL, Lin Q, Zeng ZC, et al. (2004b). Experimental study on adriamycin-induced nephrotic rats treated with Shenkangling. Zhong Guo Zhong Xi Yi Jie He Shen Bing Za Zhi 5: 574-577.

Zheng J, Wu QL, Lin Q and Zeng ZC (2005). The experimental study of the effects of the kidney Kang Ling on adriamycin induced nephropathy in rats and NF-kappa B. Fu Jian Zhong Yi Xue Yuan Xue Bao 15: 8-10.

Zheng J, Lin Q, Ai S, Chu KD, et al. (2008). Clinical study of the relationship between the NEI network and the patients with recurrent renal disease in the treatment of Chinese traditional medicine. Zhong Guo Zhong Xi Yi Jie He Shen Bing Za Zhi 9: 1000-1002.

Genetics and Molecular Research 15 (3): gmr.15038131 